\title{
Une autre maladie neurodégénérative due à l'expansion du triplet CAG : la maladie de Machado-Joseph
}

On connaît déjà cinq maladies neurodégénératives dues à une expansion instable du trinucléotide CAG l'amyotrophie spinobulbaire (CBMA), la maladie de Huntington (HD) $(\mathrm{m} / \mathrm{s}$ $n^{\circ} 4$, vol. 9, p. 488), l'ataxie spinocérébelleuse (SCA 1) $\left(m / s n^{\circ} 8-9\right.$, vol. 9, p. 1003), l'atrophie dentato-rubro-pallidoluysienne (DRPLA) $\left(\mathrm{m} / \mathrm{s} \boldsymbol{n}^{\circ} 4\right.$, vol. 10, p. 472) et le syndrome Haw River (HRS). Aujourd'hui, une nouvelle maladie vient s'ajouter à cette liste [1]. Il s'agit de la maladie de Machado-Joseph (MJD), affection neurodégénérative du système nerveux central et périphérique, transmise selon le mode autosomique dominant, de pénétrance très élevée $(98 \%)$ [2, 3]. Au niveau clinique, les patients MJD présentent une ataxie cérébelleuse, une spasticité, uneophtalmoplégie externe, une dystonie, une perturbation du mouvement des yeux, une perte sensorielle, une faiblesse musculaire et une fasciculation faciale et linguale. L'examen neuropathologique montre une perte sélective de neurones de la substance noire, du noyau dentelé du cervelet, des nerfs périphériques et des neurones des faisceaux spinocérébelleux et pyramidaux de la moelle épinière. La maladie est d'évolution progressive, plus rapide dans les formes juvéniles, et s'exprime avec une grande variabilité phénotypique, y compris à l'intérieur d'une même famille. Cette variabilité explique pourquoi, dans certains cas, il est difficile de distinguer les malades MJD de ceux présentant d'autres formes d'ataxie, telles que les ataxies spinocérébelleuses SCA-1 et SCA-2 ou la DRPIAA [4]. Initialement, la MJD avait été décrite chez des Portugais originaires de l'Archipel des Açores, où la fréquence de la maladie est de 1/4000 (île de Sào Miguel), mais depuis elle a été retrouvée dans d'autres populations, notamment au Japon où la fréquence est cependant plus faible (1/500 000). Par analyse de liaison génétique, d'abord dans des familles japonaises puis dans des familles originaires des Açores, le locus MID a été localisé sur le bras long du chromosome 14 (14q24.3q32) [5-7]. Une autre ataxie dominante, la SCA-3, a été localisée dans le même intervalle [8] ; il est donc fort probable que SCA-3 et MID soient des formes alléliques de la même maladie, tout comme la DRPLA et le HRS, tous deux caractérisés par une expansion modérée d'un triplet CAG appartenant au même gène [9-11].

I a nature de la mutation impliquée dans les cinq maladies neurodégénératives à manifestation tardive a incité une équipe japonaise à rechercher directement un gène avec une répétition CAG [1]. Cela a été réalisé par criblage d'une banque d'ADNc de cerveau humain (individu non atteint), à l'aide d'une oligosonde répétitive CTG. Ainsi, a été obtenu un clone, MJD1a, de 1776-pb avec un cadre de lecture ouvert contenant une répétition du trinucléotide CAG. Celle-ci coderait en principe pour une série de glutamines situées près de l'extrémité C-terminale de la protéine : la répétition serait située à 291 acides aminés de l'extrémité $\mathrm{N}$-terminale et à 43 acides aminés de l'extrémité C-terminale. L'ADNc identifié a été considéré comme le produit du gène responsable de la MJD car : (1) il a été localisé dans l'intervalle MJD sur le chromosome 14q, et (2) le nombre de copies CAG présentes dans le gène des individus normaux était différent de celui trouvé chez les individus atteints. En effet, dans la population japonaise, l'analyse des fragments d'ADN génomique renfermant la répétition CAG a montré que leur nombre était polymorphe : les sujets normaux possèdent entre 13 et 36 répétitions (72 allèles étudiés), alors que les patients MJD ont un allèle normal et un allèle muté avec un nombre de CAG situé entre 68 et 79 (11 des 12 malades étudiés) : on n'a pas trouvé d'allèles intermédiaires. Une fois encore, les grandes expansions sont observées chez les malades dont le début des symptômes est plus précoce (phénomène d'anticipation), cela étant plus fréquent quand l'allèle muté est transmis par le père [12]. Par ailleurs, le séquençage des allèles normaux et mutés a révélé que la répétition est interrompue par deux triplets, CAA et AAG, dans trois positions : les deux premières variations (CAA et AAG) ont été observées dans tous les allèles et la troisième (CAA) a été trouvée dans tous les allèles mutés et dans la plupart des allèles normaux. Ces interruptions, localisées dans la partie 5 ' de la répétition, indiquent que l'extrémité où l'expansion a lieu est la partie 3'. Elles semblent n'avoir aucun rôle dans la pathogénie de la maladie. Par des expériences de RT-PCR, les chercheurs ont montré que les deux allèles sont transcrits dans le cerveau des patients MJD. L'éventuelle protéine MJD la ne présente aucune homologie avec les protéines connues, à l'exception du domaine polyglutami- 


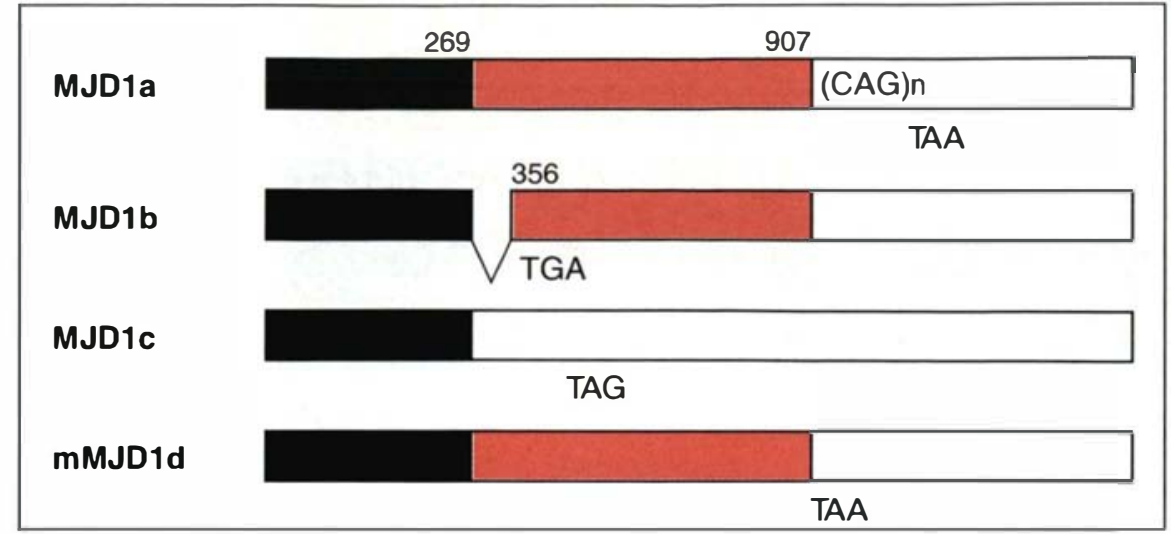

Figure 1. Les ADNc MJD1. Chez l'homme, le gène MJD1 s'exprime au moins en trois isoformes $d^{\prime} A R N m$, représentées par les clones MJD1a, b et $\mathrm{c}$, alors que chez la souris un seul messager semble exister, le mMJD1d. Les régions communes entre ces différents $A D N c$ sont colorées en noir et rouge, et les régions de séquence unique sont en blanc. Les jonctions d'épissage sont signalées par les chiffres donnant la position du nucléotide dans I'ADNc MJD1a. La fin de la région codante de chaque isoforme est indiquée approximativement par le codon de terminaison de la traduction. Parmi les quatre clones, seul MJD1a possède la répétition CAG. (D'après [1].)

ne, dont la fonction reste à déterminer.

L.es études d'hybridation avec le clone M/I)la, dépourvu de la répétition et de l'élément $A l u$ présent dans la partie 3' non codante, ont fourni des renseignements intéressants. Premièrement, l'analyse par Southern blot de l'ADN génomique digéré par différentes enzymes de restriction a toujours révélé plusieurs bandes, ce qui suggère l'existence de gènes $M / I$ ) apparentés. Ce résultat a été confirmé par criblage d'une banque d'ADN génomique humain, quatre gènes ayant été obtenus : un gène, $M / I) 1$, codant pour l'ADNc MJI)la et trois autres gènes apparentés, dénommés $M / L$ ) 2,3 et 4. Ces gènes sont localisés sur des régions chromosomiques distinctes, le $M / I) 1$ étant en $14 q 23.1$, le $M / I) 2$ en $8 q 23$, le $M / D 3$ en $14 q 21$ et $M J I) 4$ en Xp22.1 (locus STS).

Des trois gènes apparentés, seul M/L)2 présente une séquence homologue à la répétition du gène $M / I) 1$. Il s'agit de la répétition d'un hexanucléotide CAGCGG qui, s'il était inclus dans la partie codante, aurait la capacité de coder pour une série glutamine-arginine. Cependant, cette nouvelle répétition ne présente pas d'expansion, au moins dans les ADN génomiques tes-
M/I)2, 3 et 4 ont également des répétions (AG, on ne peut pas exclure leur participation dans certains cas diagnostiqués comme étant MJD. Cette idée est renforcée par le fait qu'un des douze malades japonais étudiés ne présente pas d'allèle $M / I$ )I avec l'expansion CAG. Néanmoins, les chercheurs n'ont pas écarté chez ce patient la possibilité d'une mutation ponctuelle dans le gène $M J I) 1$, ni la possibilité d'une expansion dans un autre gène, impliqué dans une autre ataxie dominante.

Récemment, une équipe canado-portugaise a rapporté le cas d'un malade présentant une forme juvénile sévère (début de la maladie à l'âge de 16 ans), issu d'une famille consanguine originaire des Açores [7, 13]. L'existence de patients homozygotes dans des isolats géographiques, comme les Açores, n'est pas étrange. A l'heure actuelle, on ne sait pas si la gravité des symptômes observés chez ce patient est due à un phénomène d'anticipation ou à la présence d'une mutation homozygote. I a MJD est une maladie dominante, s'exprimant donc chez les hétérozygotes. Dans de tels cas, les homozygotes peuvent, soit être plus gravement atteints que les hétérozygotes (par exemple dans une observation de porphyrie aiguë et dans un cas de polykystose rénale dominante), soit avoir la même symptomatologie, comme cela est attendu dans une vraie maladie dominante et a été démontré dans une observation de maladie de Huntington ( $/ \mathrm{s} \mathrm{n}^{\circ} 6$, vol. 3, p. 370). Cette dernière affection étant due, elle aussi, à une expansion de triplets codant pour des glutamines, on peut penser que la situation est similaire dans la MJD, et que la gravité des symptômes est plus liée à l'importance des répétitions qu'à l'homozygotie.

\section{RÉFÉRENCES}

1. Kawaguchi Y, Okamo o T, Taniwaki M, Airawa M, Inoue M, Katamaya S, Kawakami H, Nakamura S, Nishimura M, Akiguchi 1, Kimura J, Narumiya S, Kakizuka A. (AAC; expansions in a novel gene for Machado-Joseph disease at chromosome 14q32.1. Nature Genel $1994 ; 8$ : 221-8. 
2. Rosenberg RN. Machado-Joseph disease. Mov Disord $1992 ; 7$ : 193-203.

3. Sequeiros J, Coutinho P. Epidemiology and clinical aspects of Machado-Joseph disease. In : Advances in neurology. New York: Raven-Press, 1993: 139-53.

4. Cancel G, Dürr A, Stevanin G, Chneiweiss H, Duyckaerts C, Serdaru M, De Toffol B, Agid Y, Brice A. Is DRPLA also linked to $14 \mathrm{q}$ ? Nature Genet $1994 ; 6-8$.

5. Takiyama Y, Nishizawa M, Kawashima S, Sakamoto H, Karube Y, Shimazaki H, Soutome M, Endo K, Ohta S, Kagawa Y, Kanazawa I, Mizuno Y, Yoshida M, Yuasa T, Horikawa Y, Nagai H, Kondo T, Inuzuka T, Onodera $O$, Tsuji S. The gene for Machado-Joseph disease maps to human chromosome 14q. Nature Genet $1993 ; 4: 300-3$.

6. Sequeiros J, Silveira I, Maciel P, Coutinho P, Manala A, Gaspar C, Burlet P, Loureiro L, Guimarães J, Tanaka H, Takiyama Y, Sakamoto H, Nishhizawa M, Nomura Y, Segawa M, Tsuji S, Melki J, Munnich A. Genetic linkage studies of Machado-Joseph disease with chromosome 14q STRPs in 16 Portuguese-Azorean kindreds. Genomics $1994 ; 21: 645-8$.

7. George-Hyslop P, Rogaeva Z, Huterer J, Tsuda F, Santos J, Haines JI, Schlumpf K, Rogaev El, Liang Y, Crapper McL achlan DR,
Kennedy J, Weissenbach J, Billingsley GD, Cox DW, Lang AE, Wherrett JR. MachadoJoseph disease in pedigrees of Azorean descent is linked to chromosome 14. Am J Hum Genet $1994 ; 55: 120-5$

8. Stevanin G, Le Guern E, Ravisé N, Chneiweiss H, Dürr A, Cancel G, Vignal A, Boch AL, Ruberg M, Penet C, Pothin Y, Lagroua I, Haguenau M, Rancurel G, Weissenbach J, Agid Y, Brice A. A third locus for autosomal dominant cerebellar ataxia type I maps to chromosome 14q24.3-qter : evidence for the existence of fourth locus. $\mathrm{Am} J$ Hum Genet 1994 ; 54 : 11-20.

9. Koide R, Ikeuchi T, Onodera $O$, Tanaka $H$, Igarashi $S$, Endo $K$, Takahashi $H$, Kondo R, Ishikawa A, Hayashi T, Saito M, Tomoda A, Miike $T$, Naito $H$, lkuta $F$, Tsuji $S$. Unstable expansion of CAG repeat in hereditary dentatorubral-pallidoluysian atrophy. Nalure Genet $1994 ; 6: 9-13$.

10. Nagafuchi S, Yanagisawa $\mathrm{H}$, Sato $\mathrm{K}$, Shirayama $\mathrm{T}$, Ohsaki E, Bundo $M$, Takedo T, Tadokoro K, Kondo I, Murayzmz N, Tanaka Y, Kikishima $H$, Umino $K$, Kurosawa $H$, Furukawa T, Nihei K, Inoue T, Sano A, Komure $O$, Takahashi M, Yoshizawa T, Kanazawa I, Yamada M. Dentatorubral and pallidoluysian atrophy expansion of an unstable CAG trinucleotide on chromosome $12 \mathrm{p}$. Nature Genet $1994 ; 6: 148$.
11. Burke JR, Wingfield MS, Lewis KE, Roses $\mathrm{AD}$, Lee $\mathrm{JE}$, Hulette $\mathrm{C}$, Pericak-Vance MA, Vance JM. The Haw River syndrome : Dentatorubropallidoluysian atrophy (DRPLA) in an African-American family. Nature Genet $1994 ; 7: 5214$

12. Sequeiros J, Oliveira J, Teixeira CM, Coutinho $\mathrm{P}$, Sousa A. Anticipation of age-at-onset in Machado-Joseph disease (MJD). Am J Hum Genet 1994; 55, Suppl : A339.

13. Lang AE, Rogaeva EA, Tsuda T, Mutterer J, George-Hyslop S. Homozygous inheritance of the Machado-Joseph disease gene. Ann Neurol $1994 ; 36: 443-7$.

\section{Luisa Mota Vieira}

Inserm U. 129, 24, rue du faubourg Saint-Jacques, 75014 Paris, France.

\section{TIRÉS À PART}

I. Mota Vieira 